\title{
Hyaluronic acid-tagged silica nanoparticles in colon cancer therapy: therapeutic efficacy evaluation
}

\author{
This article was published in the following Dove Press journal: \\ International Journal of Nanomedicine \\ 12 October 2015 \\ Number of times this article has been viewed
}

Kai Liu'
Zhi-qi Wang
Shi-jiang Wang
Ping Liu ${ }^{3}$
Yue-hong Qin'
Yan Ma ${ }^{5}$
Xiao-Chen Li $^{6}$
Zhi-Jun Huo
'Department of Gastrointestinal
Surgery, ${ }^{2}$ Department of Head and
Neck Surgery, ${ }^{3}$ Department of
Radiation Oncology, Shandong Cancer
Hospital and Institute, ${ }^{4}$ Department
of Pharmacy, ${ }^{5}$ Department of
Neurosurgery, Shandong Provincial
Hospital Affiliated to Shandong
University, ${ }^{6}$ Department of Internal
Medicine, Affiliated Hospital of
Shandong Academy of Medical
Sciences, Jinan, ${ }^{7}$ Department of Breast
Disease Center, Shandong Cancer
Hospital and Institute, Jinan, People's
Republic of China

Correspondence: Zhi-Jun Huo Department of Breast Disease Center, Shandong Cancer Hospital and Institute, No 440 Jiyan Road, Jinan, Shandong 2500 I7, People's Republic of China Tel +8653 I 88382623

Fax +8653188382623

Email huozhijun7480@gmail.com

\begin{abstract}
Colon cancer is one of the leading causes of cancer-related death worldwide, and the therapeutic application of 5-fluorouracil (5-FU) is limited due to its nonspecificity, low bioavailability, and overdose. The present study is an attempt to improve the chemotherapeutic efficacy of 5-FU in colon cancers. Therefore, we have prepared 5-FU-loaded hyaluronic acid (HA)-conjugated silica nanoparticles (SiNPs) to target to colon cancer cells. In this study, we have showed the specific binding and intracellular accumulation of targeted nanoparticles based on HA surface modifications in colon carcinoma cells. The particles had spherical shapes with sizes of approximately $130 \mathrm{~nm}$. HA-conjugated nanoparticles showed a sustained release pattern for 5-FU and continuously released for 120 hours. We have further investigated the cytotoxicity potential of targeted and nontargeted nanoparticles in colo-205 cancer cells. IC50 value of 5-FU/hyaluronic acid-conjugated silica nanoparticles (HSNP) was $0.65 \mu \mathrm{g} / \mathrm{mL}$ compared with $\sim 2.8 \mu \mathrm{g} / \mathrm{mL}$ for 5 -FU/SNP after 24 hours of incubation. The result clearly showed that HA-conjugated NP was more effective in inducing apoptosis in cancer cells than nontargeted NP. The 5-FU/HSNP showed $\sim 5 \%$ of cell apoptosis (early and late apoptosis stage) compared with only $20 \%$ for 5 -FU/silica nanoparticles (SNP)-treated group. The HA-conjugated nanoparticles provide the possibility of efficient drug transport into tumors that could effectively reduce the side effects in the normal tissues. 5-FU/HSNP was highly efficient in suppressing the tumor growth in xenograft tumor model. The proportion of Ki67 in 5-FU/HSNP-treated group was significantly lower than that of either free drug or nontargeted SiNPs. Altogether, we have showed that conjugation of HA to SiNPs could result in enhanced uptake of 5-FU through CD44-mediated endocytosis uptake and could result in significant antitumor efficacy. Thus, 5-FU/HSNP could be a promising drug delivery system for colon cancer therapy.
\end{abstract}

Keywords: colon cancer, 5-fluorouracil, nanoparticles, hyaluronic acid, apoptosis

\section{Introduction}

Colon cancer is regarded as one of the most common cancers with high rate of morbidity and mortality. ${ }^{1}$ Colorectal cancer is the third most leading cause of cancerrelated death across the world, and it accounts for $40 \%$ of all cancers diagnosed every year. ${ }^{2}$ Nearly half a million cancer deaths due to colon cancers were reported in 2014 . Various treatment modalities include surgery, radiofrequency ablation, cryosurgery, chemotherapy, radiation therapy, and targeted therapy. Among all of these, chemotherapy is the most convenient and preferred treatment modality to improve patient's profile and quality of life..$^{3,4}$ Despite being a standard treatment strategy, conventional chemotherapy is far from effective, and often ineffective due to the low concentration of drug that reaches the cancer site.

In this regard, US Food and Drug Administration have approved many anticancer drugs in the treatment of colon cancers including 5-fluorouracil (5-FU), bevacizumab, 
irinotecan, capecitabine, and oxaliplatin., ${ }^{5,6}$ Among all of these, 5-FU is used as a first-line chemotherapeutic drug in colorectal cancers, and immediately after surgery, as an adjuvant therapy. In general, 5-FU is a thymidylate synthase inhibitor (interferes with DNA synthesis) that acts by arresting cancer cell growth at S-phase of cell cycle. ${ }^{7}$ Despite its potential anticancer effect, the systemic application of 5-FU is hindered by its rapid clearance from the blood circulation (in vivo). Intravenous administration of 5-FU results in a large systemic distribution with only a small fraction of the dose reaching the site of action. ${ }^{8}$ At the same time, continuous high concentration (doses) of drug can cause serious drug-related side effects. Therefore, it is utmost necessary to develop a nano drug delivery system to increase the therapeutic performance of 5-FU., ${ }^{9,10}$ To overcome the aforementioned limitations, a number of polymer-based drug delivery systems have been developed. Although polymeric nanoparticles have great potential in drug delivery, their suitability for cancer targeting remains hindered due to their poor in vivo stability and nonspecific nature. Enhanced permeation and retention effect has become a sole way of cancer targeting, wherein these nanoparticles can pass through the leaky vasculature. Studies have shown that enhanced permeation and retention effect has limited role in effective targeting of drugs to cancer sites. ${ }^{11}$ The distribution of carriers within the tumor cells is challenging due to limited permeability of NPs within the tumors and lack of inherent affinity between the passively targeted NPs and tumor cells. ${ }^{12}$ Therefore, an effective drug delivery system that can actively target the tumor and at the same time remain biocompatible needs to be developed.

Engineering multifunctional nanocarriers for targeted drug delivery shows promising potentials to revolutionize the cancer chemotherapy. Silica nanoparticles (SiNP) possesses unique advantages as a delivery carrier, including excellent biocompatibility, high hydrophobicity, systemic stability, and resistance to $\mathrm{pH}$ changes, and also, it can be largely multifunctional. ${ }^{13,14}$ Due to its nanosize, it can easily permeate the tumor tissues (via active and passive targeting) and release the drug in a sustained manner. At the same time, intracellular concentration of drug could be increased by conjugating it to a biomolecule. Hyaluronic acid (HA) is reported to possess strong affinity toward the CD44 overexpressing cancer cells. ${ }^{14}$ The HA, which is composed of $\mathrm{N}$-acetyl-D-glucosamine and D-glucuronic acid, interacts with the CDD4 overexpressing cancer cells. ${ }^{15}$ There are numerous advantages of conjugating HA on the NP surfaces: 1) CD44 receptors are overexpressed in multiple human cancer cells, and their density increases with the stages of cancers, 2) high availability and low cost, 3) HA is nonantigenic and biocompatible, and 4) high affinity toward the receptors. Therefore, HA-based targeting delivery systems are expected to show great potential in clinical applications. ${ }^{16,17}$

The main aim of the present study was to increase the therapeutic efficacy of 5-FU in colon cancers. 5-FU was encapsulated in a biocompatible silica nanocarrier and chemically conjugated with a HA biomolecule to achieve active tumor-targeting ability in CD44 overexpressed colorectal cancer cells. The anticancer ability of 5-FU-loaded HA-conjugated SiNPs was investigated by evaluating the physiochemical parameters (size, shape, and release profile), cellular uptake, cytotoxicity assay, and apoptosis analysis (qualitative and flow cytometer).

\section{Materials and methods Materials}

Aerosol-OT (AOT), vinyltrimethoxysilane, 3-aminopropyltriethoxysilane, ethyl-3-(3-dimethylamino) propyl carbodiimide hydrochloride (EDC), $N$-hydroxysuccinimide were purchased from Sigma-Aldrich Co. (St Louis, MO, USA). Sodium HA (molecular weight $=35 \mathrm{kDa}$ ) was purchased from Shandong Freda Biopharm Co., Ltd., Jinan, People's Republic of China. All other chemicals were reagent grade and used without further purifications.

\section{Preparation of 5-FU-loaded HA- conjugated SiNPs}

SiNPs were prepared by dissolving $0.45 \mathrm{~g}$ Aerosol-OT, and $800 \mu \mathrm{L}$ of 1 -butanol was added in water $(20 \mathrm{~mL})$ and constantly stirred for 30 minutes. A specified amount of $50 \mathrm{mg}$ 5-FU was first dissolved in dimethylformamide and then in the aqueous solutions. A total of $280 \mu \mathrm{L}$ of vinyltrimethoxysilane was added to the micellar system, and the solution was stirred for 60 minutes. Now, 3-aminopropyl-triethoxysilane was added to the above solution and stirred for an additional 24 hours. The 5-FU-loaded nanoparticles were centrifuged to remove the unreacted byproducts.

Before conjugation of HA to the silica NP, HA was activated by dissolving it in a HEPES buffer and stirred for 30 minutes. EDC and hydroxysuccinimide were prepared in ultra-pure water and added to the HA solution and further stirred for 60 minutes. HA-conjugated silica NP was prepared by adding three parts of activated HA solution to one part of bare NP and stirred overnight. The unreacted HA was removed by dialysis method. The amount of 5-FU entrapped in the NP was determined from the supernatant. Supernatant 
was subjected to analysis for unloaded drug concentration by HPLC. The HPLC system consisted of a Waters Model 1515 HPLC pump, a Waters autosampler Model 717 plus, and a Waters 2487 dual $\lambda$ absorbance UV detector (Waters Inc., Bedford, MA, USA). The detector wavelength was set at $260 \mathrm{~nm}$. Mobile phase of $40 \mathrm{mM}$ phosphate buffer adjusted to $\mathrm{pH} 7.0(10 \% \mathrm{w} / \mathrm{v}$ potassium hydroxide) was used in the analysis, and the flow rate was kept constant at $1 \mathrm{~mL} / \mathrm{min}$. The loading capacity (DLC) and loading efficiency (LE) were calculated using the below formulae:

$$
\begin{gathered}
\text { DLC }(w t \%)=\frac{\text { Weight of loaded drug }}{\text { Total weight of loaded drug and polymer }} \times 100 \% \\
\text { LE }(\%)=\frac{\text { Weight of loaded drug }}{\text { Weight of drug in feed }} \times 100 \%
\end{gathered}
$$

\section{Particle size analysis (DLS and TEM)}

The particle size and size distribution of NPs were evaluated by means of dynamic light scattering (DLS) instruments (Zetatrac, Microtrac Inc.). The samples were diluted suitably before the measurement. Malvern Nano-ZS 90 laser particle size analyzer was used at $25^{\circ} \mathrm{C}$. The morphological characteristics of NPs were evaluated by a transmission electron microscope (TEM, H-6009IV, Hitachi Ltd., Tokyo, Japan). The samples were diluted with distilled water and put on a copper grid covered with carbon. The samples were then negatively stained with $2 \%$ phosphotungstic acid and dried at room temperature.

\section{Drug release study}

The release profile of 5-FU from the silica NP system was characterized by dialysis method. The experiments were carried out in phosphate-buffered saline (PBS) of pH 7.4 at $37^{\circ} \mathrm{C}$. The release study was conducted in sink conditions. Specified quantity of dried NP (1 mg of equivalent 5-FU) was dispersed in $1 \mathrm{~mL}$ of distilled water and transferred to dialysis tubes and kept in a vessel containing PBS (volume of dialysate $=30 \mathrm{~mL}$ ) of $\mathrm{pH}$ 7.4. The whole system was incubated at $37^{\circ} \mathrm{C}$ under shaking. At specific time intervals, $1 \mathrm{~mL}$ of release medium was removed and analyzed for amount of 5-FU released. Release media was subjected to analysis for drug concentration by HPLC. All the experiments were repeated thrice, and triplicates were analyzed in each set.

\section{Cytotoxicity assay}

The human colon carcinoma (colo-205) was cultured in RPMI supplied with 10\% FBS and antibiotics (50 units $/ \mathrm{mL}$ penicillin and 50 units $/ \mathrm{mL}$ streptomycin) at $37^{\circ} \mathrm{C}$ in a humidified atmosphere containing $5 \% \mathrm{CO}_{2}$. No ethics approval was required for the use of this cell line. The cytotoxicity potential of individual formulations was evaluated by means of MTT colorimetric assay. Cells $\left(1 \times 10^{4}\right.$ per well $)$ were seeded in a flat-bottomed 96 -well plate and incubated at $37^{\circ} \mathrm{C}$ and in $5 \%$ $\mathrm{CO}_{2}$. Cells were then exposed to a series of blank NPS and drug-loaded formulations at various dose concentrations. The cells were incubated for 24 hours and 48 hours, respectively. Cells were then treated with MTT reagent $(20 \mu \mathrm{L} /$ well volume from $5 \mathrm{mg} / \mathrm{mL}$ solution in PBS) for 3 hours at $37^{\circ} \mathrm{C}$. DMSO $(100 \mu \mathrm{L})$ was added to each well to dissolve the formazan crystals. The optical density (OD) was recorded at $570 \mathrm{~nm}$ in a microplate reader, and percentage of residual cell viability was determined. The cell cytotoxicity of different formulations is defined as the relative viability, which is the ratio of the number of live cells to that of the control cells (100\%).

\section{Cellular uptake study}

The cellular uptake of targeted (HA-conjugated) and nontargeted NP (silica NP) was studied on colo-205 cancer cells in a time-dependent manner. Rhodamine-B was used as a fluorescent dye and loaded in the NP. The cells were exposed with $1 \mathrm{mg} / \mathrm{mL}$ of Rho-B/SNP and Rho-B/hyaluronic acidconjugated silica nanoparticles (HSNP) and incubated for various time points in standard $37^{\circ} \mathrm{C}$ and $5 \% \mathrm{CO}_{2}$ conditions. The cells were then washed with cold PBS three times and completely dissolved in DMSO. The uptake of nanoparticles was measured using a fluorescence plate reader (Beckman Coulter, Inc., Brea, CA, USA). The total amount of NP entered into the cancer cell was quantified using the below formula:

$$
\underset{\text { uptake }}{\% \text { cellular }}=\frac{\text { Amount of fluorescent dye taken up by cells }}{\text { Total amount of fluorescent dye added }} \times 100
$$

\section{Confocal laser scanning microscopy study}

The cancer cells were seeded in a six-well culture plate and incubated for 24 hours. The cells were then treated with Rho-B/HSNP for 1 hour in serum-free RPMI media. The cells were washed three times with PBS to remove unbound nanoparticles and fixed in 4\% paraformaldehyde. Lysotracker Green was used to stain the lysosomal region of the cancer cells. Fluorescence micrographs were acquired using the DAPI filter set of Nikon Eclipse TS100-F.

\section{Apoptosis analysis (Hoechst 33382 staining)}

The cytotoxic effect of individual formulations was further characterized by apoptosis analysis. To determine the apoptosis effect, cells were seeded and incubated for 
24 hours. Cells were then exposed with $1 \mu \mathrm{g} / \mathrm{mL}$ of free 5-FU, 5-FU/SNP, and 5-FU/HSNP and incubated for further 24 hours. The cells were washed twice and fixed with 4\% paraformaldehyde for 20 minutes, and nuclei were stained with Hoechst 33342 ( $5 \mathrm{mg} / \mathrm{mL}$ ) for 10 minutes at room temperature. After rinsing with PBS for three times, the images of cells were directly captured by fluorescent microscope (Nikon Eclipse TS100-F).

\section{Cellular apoptosis analysis}

The apoptosis analysis was performed by flow cytometer after staining with Annexin V-Fluorescein Isothiocyanate Apoptosis Detection Kit I (BD Biosciences, San Jose, CA, USA) according to the manufacturer's instructions. A549 cells were seeded in a six-well plate $\left(1 \times 10^{6}\right.$ cells/well $)$ and allowed to attach for 24 hours. Next day, media was removed and replaced with fresh media containing free 5-FU, 5-FU/ SNP, and 5-FU/HSNP. The formulations were incubated for 24 hours. Cells were stained with Annexin V-fluorescein isothiocyanate and propidium iodide; the percentage of apoptotic cells was quantified by fluorescence-activated cell sorting analysis.

\section{Antitumor efficacy study}

The animal study and protocols were approved (2015JUH45LK12) by the Institutional Animal Care and Use Committee, Shandong Cancer Hospital and Institute, People's Republic of China. A 12 hour-day/night cycle was followed, and animals were given free access to food and water. The antitumor efficacy study was performed in colon cancer cell bearing xenograft tumor model. The tumor model was established as per the reported protocol. Briefly, $1 \times 10^{6}$ colo-205 cells (in $100 \mu \mathrm{L}$ PBS) were subcutaneously injected into the right flank of mice, and the tumors were allowed to grow until $100 \mathrm{~mm}^{3}$. The mice were randomly divided into four groups and injected with respective formulations. The tumor size was measured using calipers. The tumor volume was calculated using the formula $V=1 / 2 \times a \times b^{2}$, where $V$ is the tumor volume, and $\mathrm{a}$ and $\mathrm{b}$ are the largest and smallest diameters of the tumors. The mice were sacrificed, and tumors were extracted and histological and immunohistochemical studies were performed.

\section{Statistical analysis}

Data were expressed as the mean value \pm SD. Statistical analysis was performed with one-way analysis of variance (ANOVA) using SPSS software. $P$-value less than 0.05 were considered to be statistically significant.

\section{Results and discussion Preparation and characterization of 5-FU-loaded HA-SiNP}

Colon cancer is regarded as one of the most common cancers with high rate of morbidity and mortality. In this regard, chemotherapy is the most convenient and preferred treatment modality to improve patient's profile and quality of life. 5-FU is used as a first-line chemotherapeutic drug in colorectal cancers, and immediately after surgery as an adjuvant therapy. 5-FU is a thymidylate synthase inhibitor (interferes DNA synthesis), which acts by arresting cancer cell growth at S-phase of cell cycle. However, intravenous administration of 5-FU results in large systemic distribution, with only a small fraction of the dose reaching the site of action. Therefore, it is utmost necessary to develop a nano drug delivery system to increase the therapeutic performance of 5-FU. In the present study, SiNP was used owing to its unique advantages such as excellent biocompatibility, high hydrophobicity, systemic stability, and resistant to $\mathrm{pH}$ changes, and also, it could be largely multifunctional. Furthermore, HA was conjugated on the surface of SiNP. Before conjugation of HA to the silica NP, HA was activated to expose free carboxylic acid group. The carboxylic group of HA was conjugated with the amine functional group of silica (Figure 1). The HA, which is composed of $\mathrm{N}$-acetyl-D-glucosamine and D-glucuronic acid, interacts with the CDD4 overexpressing cancer cells. HA-based targeting delivery systems are expected to show great potential in clinical applications. The focus of this work was to use HA-SiNP to encapsulate 5-FU and deliver to colon cancer cells.

\section{Particle size distribution and morphology of 5-FU-loaded NP}

HA-SiNP was applied in order to improve the anticancer efficacy of 5-FU and to reduce its associated side effects. 5-FU was efficiently loaded into the core of Si NP with a high loading efficiency. The loading efficiency of 5-FU in NP was observed to be $>90 \%$ with a high loading capacity of $>15 \%$. The conjugation of HA to the SiNP did not alter the loading efficiency of 5-FU. The average particle size of SNP and HSNP was observed to be $\sim 95 \pm 2.3 \mathrm{~nm}$ and $138 \pm 3.4 \mathrm{~nm}$, respectively (Figure $2 \mathrm{~A}$ ). The polydispersity index represented a uniform mono-dispersion with PDI $\sim 0.12$. It has been well documented that particles of $<200 \mathrm{~nm}$ are more advantageous as nanoparticles of reduced particle size could effectively increase the blood circulation profile of anticancer drugs and thereby avoid the reticuloendothelial system-mediated systemic clearance and passively target the anticancer drug to the tumor tissues. ${ }^{18}$ 

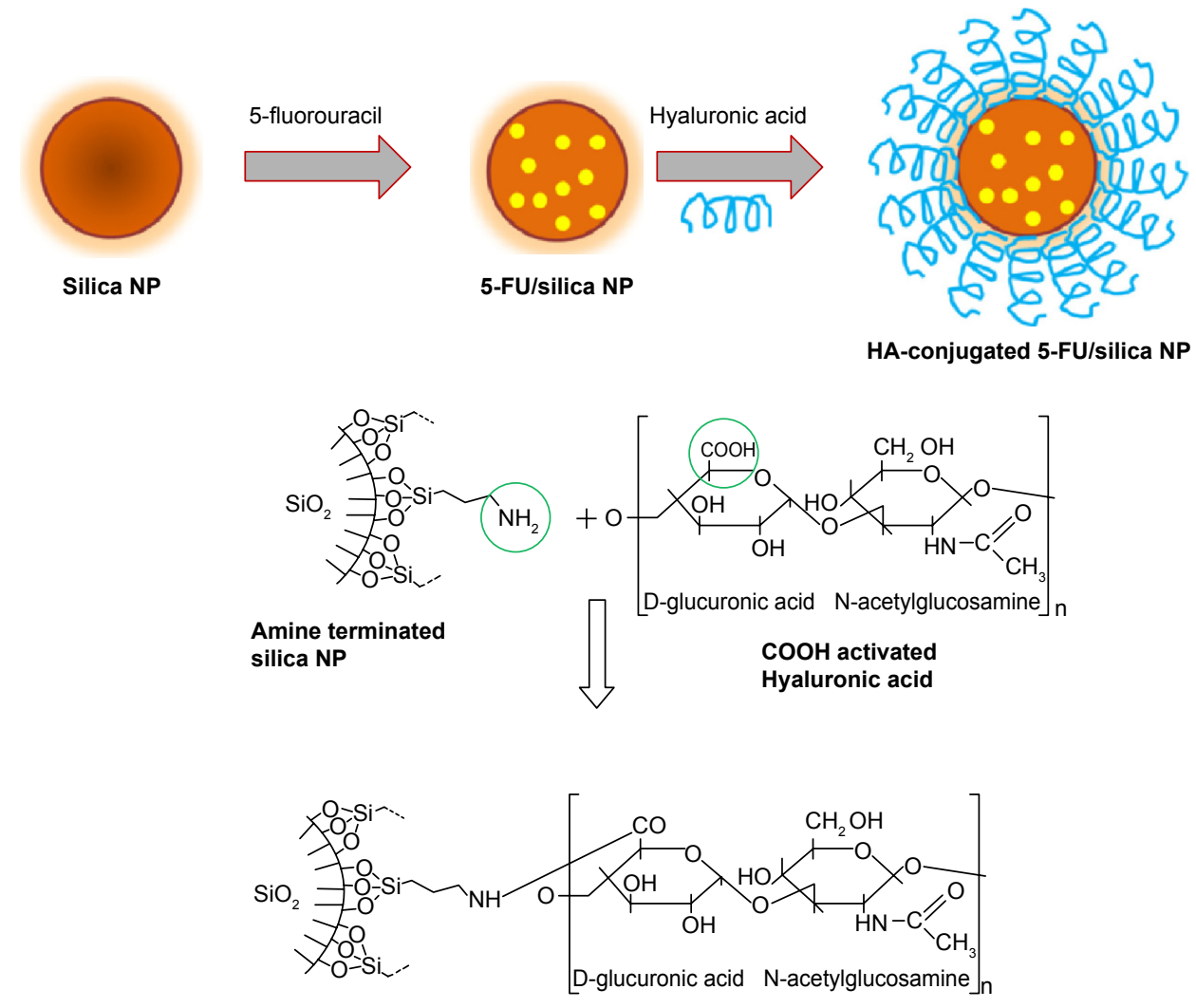

Hyaluronic acid-conjugated silica NP

Figure I Schematic illustration of preparation of hyaluronic acid-conjugated silica nanoparticles.

Notes: 5-fluorouracil was used as a model drug to load in the nanoparticles. The carboxylic group of HA was activated and conjugated with the amine functional group of silica nanoparticles.

Abbreviations: HA, hyaluronic acid; 5-FU, 5-fluorouracil; NP, nanoparticles.

The HSNP showed perfect spherical particles, which were uniformly dispersed in the TEM grid (Figure 2B). A darker core in the center and relatively grayish brush on the surface represent the HA-conjugated SiNPs. The hydrophobic drug is expected to locate at the center of the core. The particles of HSNP were stable and homogeneous, and the diameter was in good agreement with the results of particle size from DLS analysis.

\section{Drug release study}

The in vitro release study of 5-FU/SNP and 5-FU/HSNP was performed in $\mathrm{PBS}(\mathrm{pH} 7.4)$ at $37^{\circ} \mathrm{C}$ (Figure 3). The data
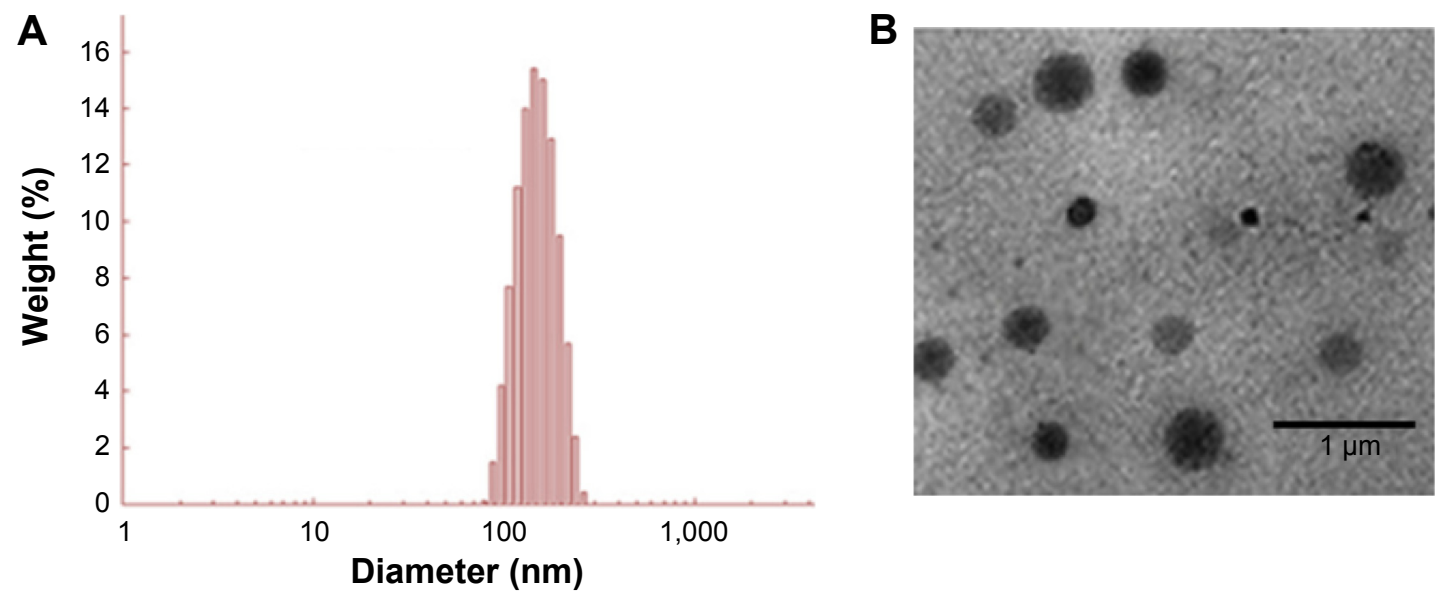

Figure 2 Physicochemical characterization of 5-FU/HSNP.

Notes: (A) Particle size distribution of 5-FU/HSNP, (B) transmission electron microscope images of 5-FU/HSNP.

Abbreviations: 5-FU, 5-fluorouracil; HSNP, hyaluronic acid-conjugated silica nanoparticles. 


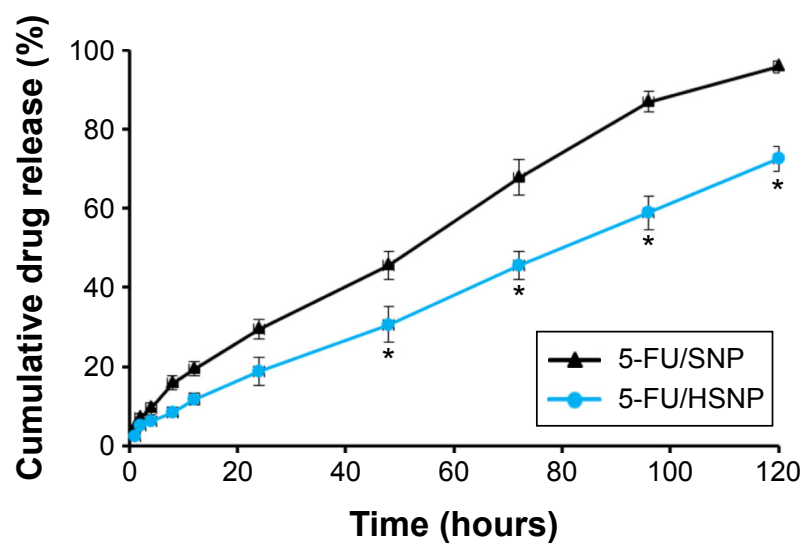

Figure 3 In vitro release profile of 5-FU/SNP and 5-FU/HSNP in phosphate-buffered saline $(\mathrm{pH}=7.4)$ at $37^{\circ} \mathrm{C}$.

Notes: The release study was conducted up to 120 hours, and the amount of drug released was evaluated by means of HPLC technique. $* P<0.05$.

Abbreviations: HPLC, high performance liquid chromatography; 5-FU, 5-fluorouracil; HSNP, hyaluronic acid-conjugated silica nanoparticles; SNP, silica nanoparticles.

suggested that the rate of 5-FU release was significantly slower in HA-conjugated nanoparticle than SiNPs. It can be clearly seen that nearly $\sim 30 \%$ of drug was released from SNP, whereas only $\sim 18 \%$ of drug was released from HSNP in 24 hours. The trend continued until the end of release study wherein nearly $100 \%$ of drug released from SNP compared with $\sim 70 \%$ from HSNP. The difference in drug release between the two carriers could be due to the presence of HA on the surface of nanoparticles. The delayed release of drug from HSNP indicates that it can act as an efficient drug carrier and could minimize the exposure of chemotherapeutic agents to normal tissues and increase the accumulation in tumor tissues. ${ }^{19}$

It could be safely expected that 5-FU could be slowly released in plasma (limited release) under normal physiological conditions ( $\mathrm{pH}=7.4)$, while quickly released at the solid tumor site $(\mathrm{pH}=5.5)$. The distinctive different release behaviors of 5-FU/SNP and 5-FU/HSNP indicated that drugs encapsulated in HA-conjugated nanoparticles could release in circulation at a stable speed. Thus, the drug concentration in plasma could be kept at a relatively stable level to guarantee that enough amount of drug would reach the tumor tissues. ${ }^{20}$

\section{Cellular uptake of nanoparticles}

5-FU was replaced with rhodamine-B to investigate the cellular uptake potential of targeted and nontargeted nanoparticles. The specific binding affinity of HA toward the CD44 overexpressing colon cancer cell was evaluated by means of cellular uptake study. As seen from Figure 4A, colo-205 cells incubated with SNP and HSNP showed remarkable uptake in a time-dependent manner. Specifically, HA-conjugated NP showed a significantly higher uptake throughout the study period. The variations in the uptake of different formulations could have occurred due to the different mechanisms of uptake. The SNP could be internalized due to the simple energy-dependent process via endocytosis receptor, whereas

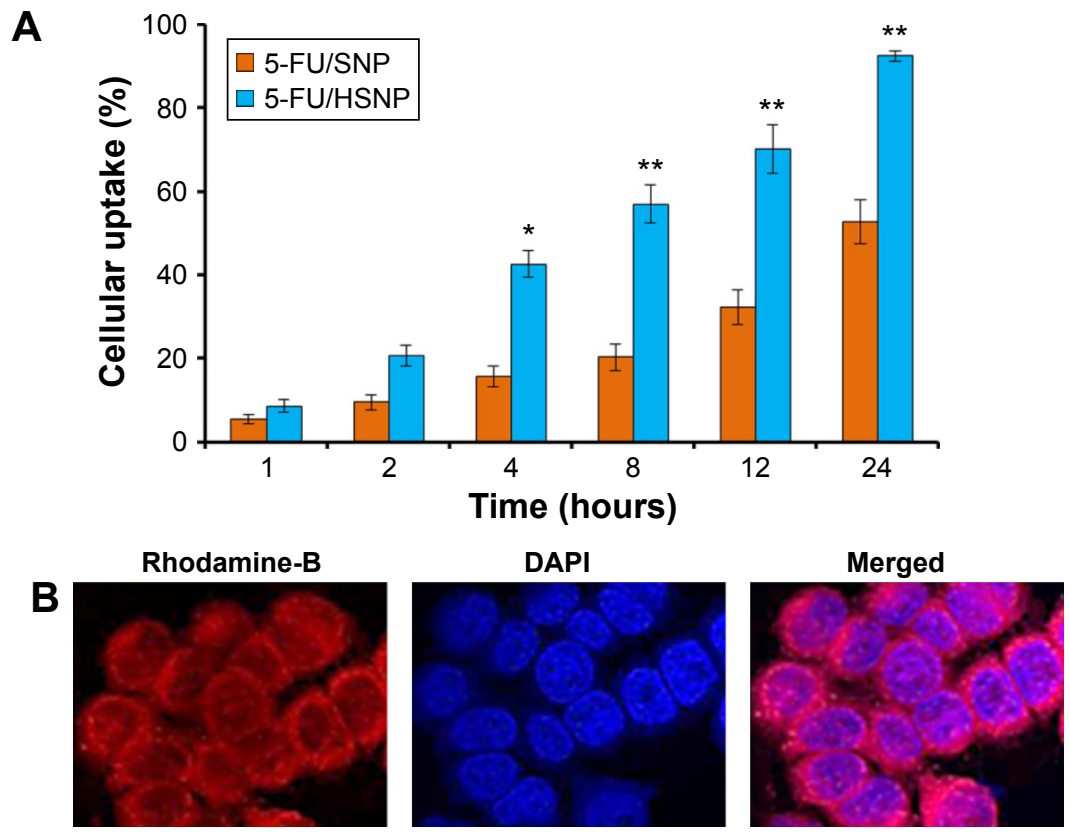

Figure 4 Cellular uptake of targeted and non-targeted nanoparticles.

Notes: (A) Intracellular uptake of 5-FU/SNP and 5-FU/HSNP in colo-205 colon cancer cells. Rhodamine-B was used as a fluorescent dye. The uptake is shown as a percentage of total amounts of NP (dye) incubated with the cancer cells. (B) Representative confocal microscopy images of 5-FU/HSNP in colo-205 cancer cells. The cells are stained with Lysotracker lysosomal stain, and DAPI was used to stain the nucleus. ${ }^{*} P<0.05, * * P<0.0$ I.

Abbreviations: 5-FU, 5-fluorouracil; HSNP, hyaluronic acid-conjugated silica nanoparticles; SNP, silica nanoparticles; NP, nanoparticle. 
HSNP could be internalized by the specific CD44-mediated cellular pathways. The influence of targeting ligand on the NP surface was clearly visible after 4 hours of incubation, wherein HSNP exhibited $>45 \%$ of cellular internalization compared with $20 \%$ for nontargeted NP. The trend continued up to 24 hours. These results are consistent with the previous observation wherein it has been shown that HA coating enhances the permeability of 5-FU containing nanoparticles by a receptor-mediated internalization. ${ }^{21}$ From the results, it is apparent that the actively targeted nanoparticles will deliver more anticancer agent to cancer cell than the nontargeted one. These data suggest a role for receptor-mediated internalization of the actively targeted HSNP.

The mechanism of cellular uptake was further confirmed by confocal laser scanning microscopy. In the present study, nuclei were stained with Hochest33342 and the nanoparticle was loaded with rhodamine-B (Figure 4B). It could be easily seen that cytoplasm showed a bright red fluorescence, whereas nucleus was in blue color after 1 hour of incubation time. It should be noted that no fluorescence was observed in the nucleus and much of fluorescence was located in the cytoplasmic region. The nanoparticle formulation is expected to destabilize and disassemble in the lysosomal compartment to allow 5-FU release, which will then travel to nucleus and perinucleus region and enhance the anticancer efficacy. Similar mechanisms have been recently reported for a number of ligand decorated drug-loaded block copolymer micelle formulations targeted to relevant cancer biomarkers like EGFR, folate receptor, and integrin $\alpha \mathrm{V} \beta 3$.

\section{In vitro cytotoxicity assay}

The cytotoxicity of blank nanoparticle was investigated in Colo-205 colon cancer cells. The cells were treated with individual formulations at various concentrations and incubated for 24 hours. As seen from Figure 5A, blank NP did not induce any appreciable toxicity, and the cell viability remained more than $85 \%$ throughout all the concentrations tested. The high cell viability of blank NP indicates its excellent biocompatibility and would be suitable for the systemic administration or cancer targeting. It is worth noting that at highest concentration $(100 \mu \mathrm{g} / \mathrm{mL})$, bare SNP exhibited $\sim 85 \%$ cell viability, whereas HA-conjugation on the surface significantly improved the cell viability indicating its excellent biocompatibility.

The cytotoxic effect of free 5-FU, 5-FU/SNP, and 5-FU/HSNP was evaluated in colo-205 colon cancer cells
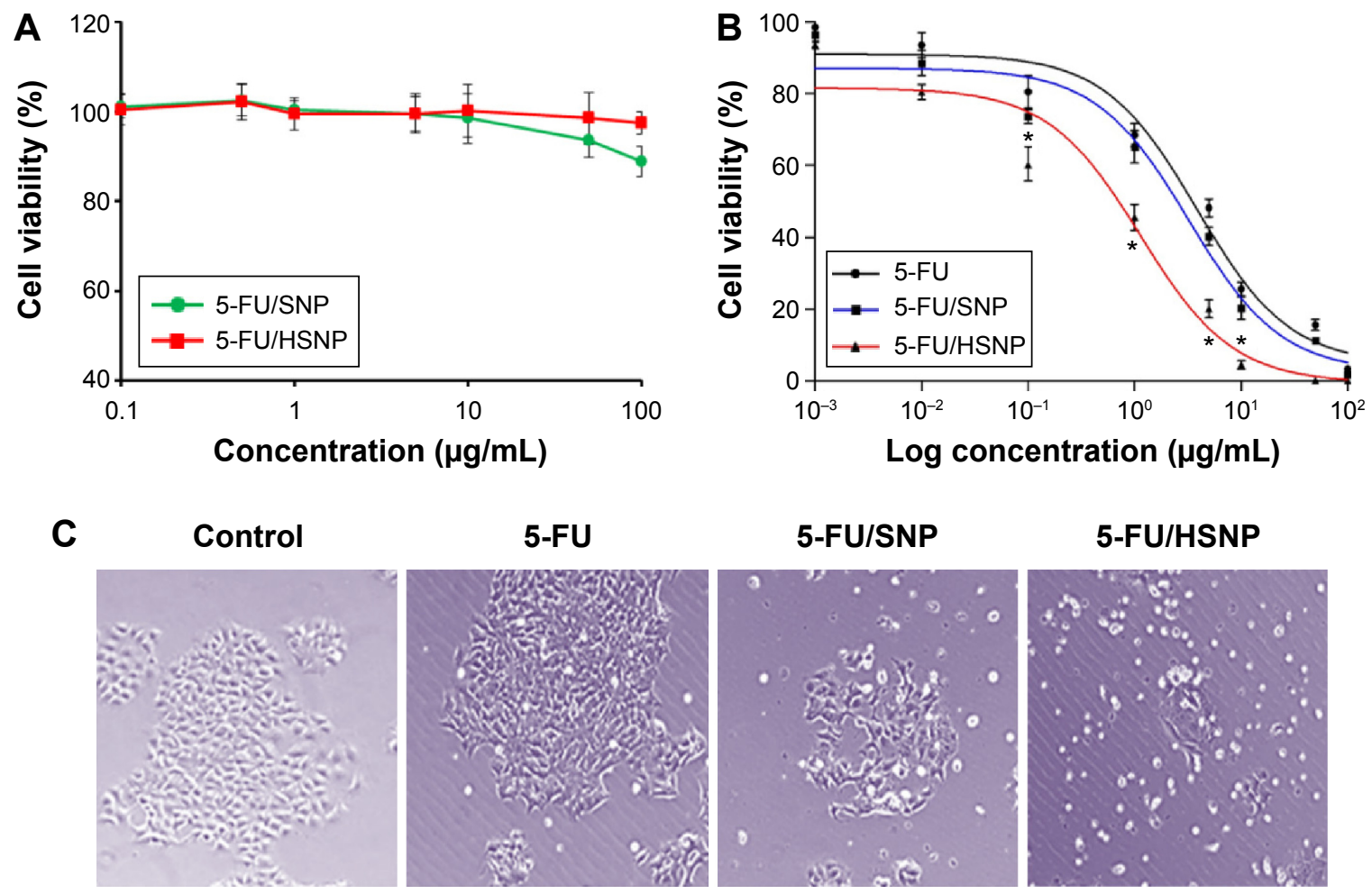

Figure 5 Cytotoxicity assay.

Notes: (A) The toxicity of blank nanoparticles on colo-205 cancer cells, (B) cytotoxicity of 5-FU, 5-FU/SNP, and 5-FU/HSNP in colo-205 colon cancer cells. All experiments were done by MTT assay in four replicates from three independent experiments. Data was represented as mean \pm SD. (C) Morphological images of cancer cells upon treatment with the respective formulations. ${ }^{*} P<0.05$.

Abbreviations: 5-FU, 5-fluorouracil; HSNP, hyaluronic acid-conjugated silica nanoparticles; SNP, silica nanoparticles; NP, nanoparticles. 
(Figure 5B and C). The cell viability of cancer cells was determined using MTT assay. The result clearly showed a dose-dependent cytotoxic effect of all the formulations. Especially, HSNP showed a superior anticancer effect after 24 hours of incubation. For example, IC50 value of 5-FU/ HSNP was $0.65 \mu \mathrm{g} / \mathrm{mL}$ compared with $\sim 2.8 \mu \mathrm{g} / \mathrm{mL}$ for 5-FU/SNP after 24 hours incubation. These observations well correlated with the higher accumulation of drug in the cancer cells due to the enhanced uptake of HA-conjugated nanoparticles. These results are consistent with other studies wherein it has been shown that HA-conjugated nanomaterials have good tumor-targeting and penetrating ability for drug delivery under in vivo and in vitro models. ${ }^{22}$

Specific targeting of malignant tissue for diagnosis and therapy is one of the major goals in recent nanotechnology. The use of specific modified nanoparticles offers the possibility of efficient drug transport and application into the targeted tissue. Therefore, HSNP could be an interesting carrier for the successful delivery of 5-FU in colon cancers.

\section{Qualitative apoptosis analysis (Hoechst staining)}

The cytotoxic effect of individual formulation was studied by Hoechst staining and visualized by DM6000CS, Leica Microsystems, Wetzlar, Germany. Untreated cells were homogeneously tarnished blue, with few or no apoptotic cells existed as shown in Figure 6. The control cells maintained a definite morphology with clear boundary. The exposure of free drug slightly fragmented the cancer cells; however, remarkable apoptosis was observed in 5-FU/HSNP-treated cancer cell group. The 5-FU/HSNP-treated group showed highly condensed cell, had thick-stained nuclei, and had fragmented chromatin and apoptotic bodies. The qualitative apoptosis study was consistent with the cytotoxicity study in which HA-conjugated nanoparticles showed a superior anticancer activity.

\section{Quantitative apoptosis analysis (flow cytometer)}

The quantitative apoptosis analysis was investigated by Annexin V-FITC/PI staining assay. Annexin V/FITC detects the phosphatidylserine externalization, which characterizes early apoptotic events. Cancer cell death can be programed (apoptosis) or nonprogramed (necrosis). The apoptosisinducing effect of formulations was evaluated by counting the early apoptosis and late apoptosis (Figure 7). The result clearly showed that HA-conjugated NP was more effective in inducing apoptosis in cancer cells than nontargeted NP. For example, 5-FU/HSNP showed $\sim 45 \%$ of cell apoptosis (early and late apoptosis stage) compared with only $20 \%$ for 5-FU/SNP-treated group. The result was consistent with the cellular uptake study, where HA-conjugated NP showed an enhanced cellular uptake. 5-FU, being a pyrimidine analogue, gets metabolized into different cytotoxic metabolites such as fluorodeoxyuridine monophosphate (FdUMP), fluoro-deoxyuridine triphosphate (FdUTP), and fluorouridine triphosphate (FUTP) inside the cells. These derivatives incorporate into DNA and RNA leading to cell death. One of the cytotoxic metabolites, FdUMP, blocks the synthesis of pyrimidine thymidine, which is required for DNA replication. The effectiveness of HSNP has been successfully proved in our study, which makes HA-conjugated NP a promising source for colon cancer therapy with high antitumor activity.

\section{Antitumor efficacy analysis}

The antitumor efficacy of individual formulations was tested in xenograft tumor model. As shown in Figure 8A, tumor volume constantly increased in case of untreated mice group. The free drug, though inhibited the progression of tumor to an extent, is far behind the reasonable antitumor response. Consistent with the in vitro studies, HSNP was highly efficient in suppressing the tumor growth. The remarkable
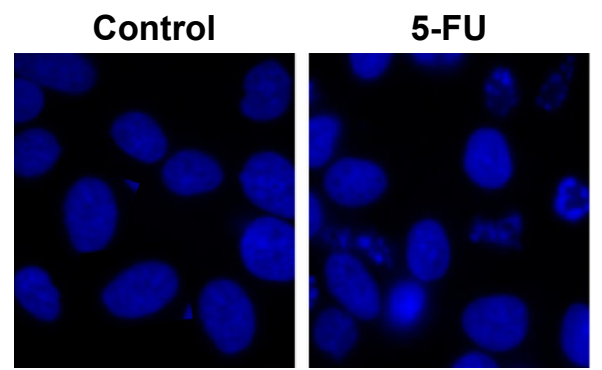

5-FU/SNP

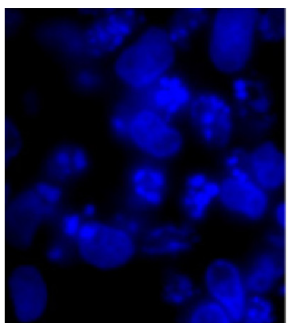

5-FU/HSNP

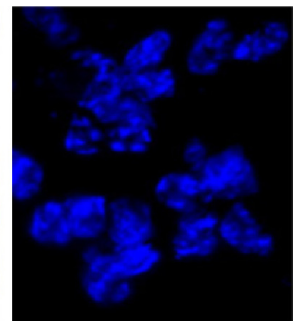

Figure 6 Cell apoptosis observed by Hoechst 33342 staining.

Notes: Cells were treated with 5-FU, 5-FU/SNP, and 5-FU/HSNP for 24 hours, subsequently stained, and observed under UV light or white light. Abbreviations: 5-FU, 5-fluorouracil; HSNP, hyaluronic acid-conjugated silica nanoparticles; SNP, silica nanoparticles; NP, nanoparticles. 


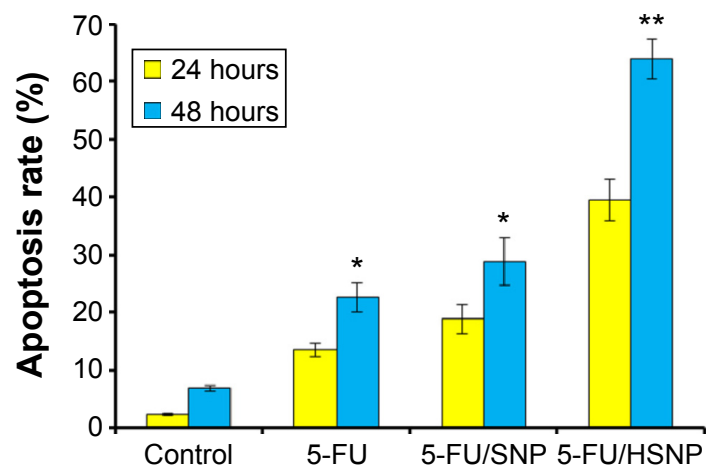

Figure 7 Annexin V-FITC FACS apoptosis analysis of HN6 cancer cells after treatment with free 5-FU, 5-FU/SNP, and 5-FU/HSNP for 24 hours and 48 hours. Notes: $* P<0.05, * * P<0.01$.

Abbreviations: FACS, fluorescence-activated cell sorting; 5-FU, 5-fluorouracil; HSNP, hyaluronic acid-conjugated silica nanoparticles; SNP, silica nanoparticles.

antitumor response might be attributed to the targeting ability of HA, which is present on the surface of nanoparticles. The targeting moiety could enhance the concentration of 5-FU within the tumor tissues.

\section{Histological and immunohistochemical analysis}

H\&E staining clearly showed that 5-FU/HSNP exhibits typical signs of cancer cell apoptosis such as apoptotic body formation and could inhibit the cancer cell proliferation (Figure 8B). Similar results were observed after Ki67 antibody staining. 5-FU/HSNP showed remarkable anticancer activity in colon cancer cells (Figure 8C). The proportion of Ki67 in 5-FU/HSNP-treated group was significantly lower than that of either free drug or nontargeted SiNPs.

\section{Conclusion}

In conclusion, we have successfully prepared 5-FU-loaded HA-conjugated SiNPs to target the colon cancer cells. In this study, we have showed the specific binding and intracellular accumulation of targeted nanoparticles based on HA surface modifications in colon carcinoma cells. The particles had spherical shapes with sizes of approximately $130 \mathrm{~nm}$.
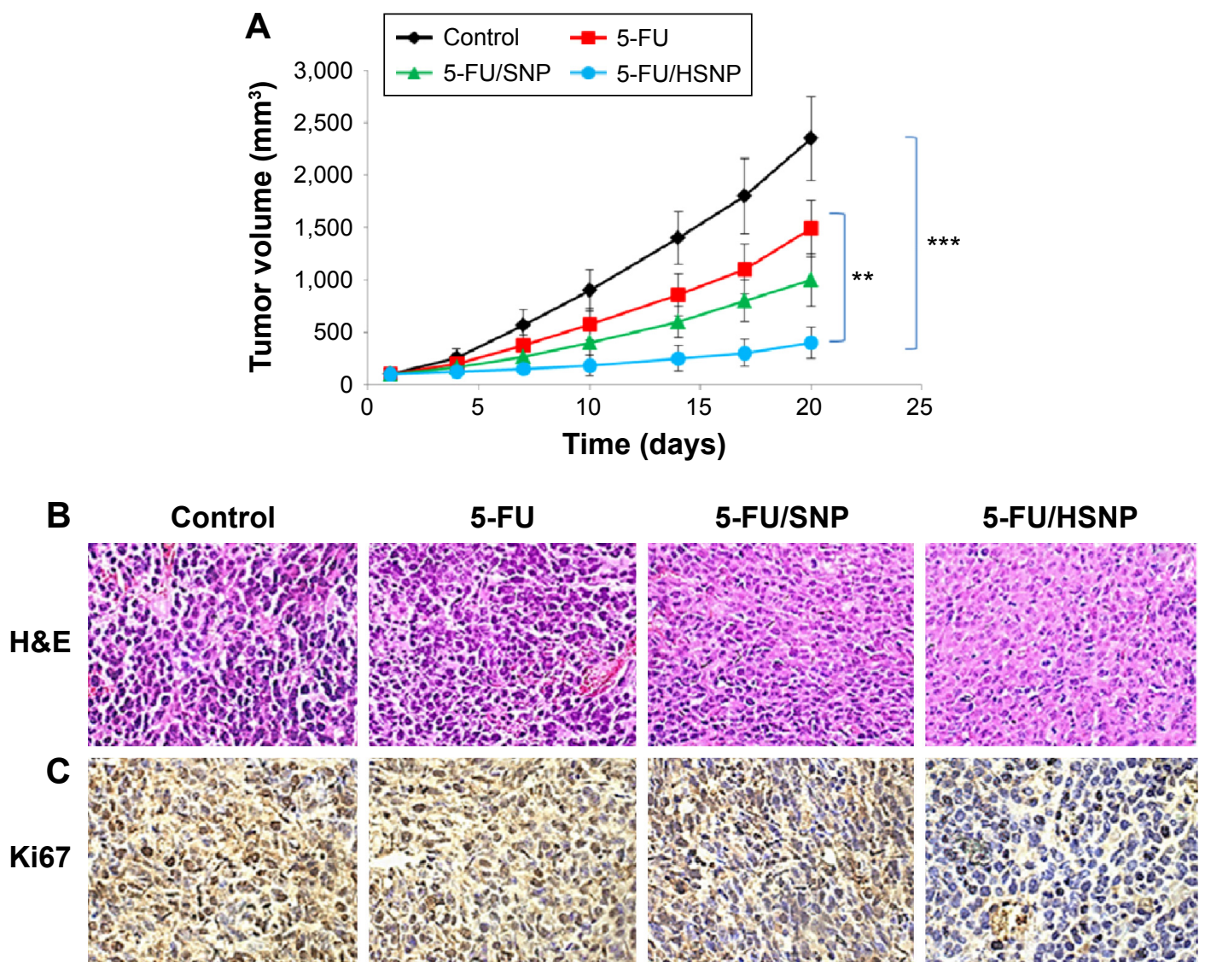

Figure 8 Antitumor efficacy of different formulations.

Notes: (A) Tumor inhibitory effect of different formulations on colon cancer cell bearing xenograft tumor model. The antitumor efficacy study was carried up to 20 days. (B) H\&E staining. (C) Ki67 immunohistochemical study in extracted tumor. H\&E staining: Eosin is an acidic dye, and it stains basic (or acidophilic) structures red or pink. Hematoxylin can be considered as a basic dye (general formula for basic dyes is dye $\mathrm{Cl}^{-}$). Hematoxylin is actually a dye called hematein (obtained from the log-wood tree) used in combination with aluminium ions $\left(\mathrm{Al}^{3+}\right) . \mathrm{Ki}-67$ is a protein in cells that increases as they prepare to divide into new cells. A staining process can measure the percentage of tumor cells that are positive for $\mathrm{Ki}-67$. The more positive cells are present, the more quickly they are dividing and forming new cells and more densely they are stained. $* * P<0.0 \mathrm{I}, * * * P<0.00 \mathrm{I}$. Abbreviations: 5-FU, 5-fluorouracil; HSNP, hyaluronic acid-conjugated silica nanoparticles; SNP, silica nanoparticles. 
HA-conjugated nanoparticles showed a sustained release pattern for 5-FU and continuously released for 120 hours. We have further investigated the cytotoxicity potential of targeted and nontargeted nanoparticles in colo-205 cancer cells. IC50 value of 5-FU/HSNP was $0.65 \mu \mathrm{g} / \mathrm{mL}$ compared with $\sim 2.8 \mu \mathrm{g} / \mathrm{mL}$ for 5 -FU/SNP after 24 hours of incubation. The result clearly showed that HA-conjugated NP was more effective in inducing apoptosis in cancer cells than nontargeted NP. The 5 -FU/HSNP showed $\sim 45 \%$ of cell apoptosis (early and late apoptosis stage) compared with only $20 \%$ for 5-FU/SNP-treated group. The HA-conjugated nanoparticles provide the possibility of efficient drug transport into tumors that could effectively reduce the side effects in the normal tissues. 5-FU/HSNP was highly efficient in suppressing the tumor growth in xenograft tumor model. The proportion of Ki67 in 5-FU/HSNP-treated group was significantly lower than that of either free drug or nontargeted SiNPs. Altogether, we have showed that conjugation of HA to SiNPs could result in enhanced uptake of 5-FU through CD44-mediated endocytosis uptake and could result in significant antitumor efficacy. Thus, 5-FU/HSNP could be a promising drug delivery system for colon cancer therapy.

\section{Acknowledgment}

This work was supported by the Traditional Chinese Medicine Science and Technology Development Plan of Shandong Province (No 2013-210).

\section{Disclosure}

The authors report no conflicts of interest in this work.

\section{References}

1. American Cancer Society. Colorectal Cancer Facts and Figures 2011-2013. Atlanta, GA: American Cancer Society; 2011.

2. World Health Organization. Colorectal Cancer: Estimated Incidence, Mortality and Prevalence Worldwide in 2012. Available from: http:// globocan.iarc.fr/old/FactSheets/cancers/colorectal-new.asp. Accessed August 28, 2015.

3. Jemal A, SiegelR, WardE, etal.Cancer statistics. CA Cancer JClin. 2006;56: 106-130.

4. Kanwar JR, Roy K, Kanwar RK. Colon cancer genetics, nanotherapy and individualized chemotherapy. In: Zhou S, Sneed KB, Whelan G, Pathak YV, editors. Clinical and Translational Pharmacogenomics. Boca Raton, FL: CRC Press; 2014.

International Journal of Nanomedicine

\section{Publish your work in this journal}

The International Journal of Nanomedicine is an international, peerreviewed journal focusing on the application of nanotechnology in diagnostics, therapeutics, and drug delivery systems throughout the biomedical field. This journal is indexed on PubMed Central, MedLine, CAS, SciSearch $\AA$, Current Contents $\AA /$ Clinical Medicine,
5. Glehen O, Kwiatkowski F, Sugarbaker PH, et al. Cytoreductive surgery combined with perioperative intraperitoneal chemotherapy for the management of peritoneal carcinomatosis from colorectal cancer: a multi-institutional study. J Clin Oncol. 2004;22:3284-3292.

6. Cohen MH, Gootenberg J, Keegan P, Pazdur R. FDA drug approval summary: bevacizumab plus FOLFOX4 as second-line treatment of colorectal cancer. Oncologist. 2007;12:356-361.

7. Wigmore PM, Mustafa S, El-Beltagy M, Lyons L, Umka J, Bennett G. Effects of 5-FU. Adv Exp Med Biol. 2010;678:157-164.

8. de Gramont A, Figer A, Seymour M, et al. Leucovorin and fluorouracil with or without oxaliplatin as first-line treatment in advanced colorectal cancer. J Clin Oncol. 2000;18:2938-2947.

9. Longley DB, Harkin DP, Johnston PG. 5-Fluorouracil: mechanisms of action and clinical strategies. Nat Rev Cancer. 2003;3:330-338.

10. Klotz HP, Weder W, Largiader F. Local and systemic toxicity of intra hepaticarterial 5-FU and high-dose or low-dose leucovorin for liver metastases of colorectal cancer. Surg Oncol. 1994;3:11-16.

11. Aslan B, Ozpolat B, Sood AK, Lopez-Berestein G. Nanotechnology in cancer therapy. J Drug Target. 2013;10:904-913.

12. Lammers T, Kiessling F, Hennink WE, Storm G. Drug targeting to tumors: principles, pitfalls and (pre-) clinical progress. J Control Release. 2012;161: 175-187.

13. Roy I, Ohulchanskyy TY, Pudavar HE, et al. Ceramic-based nanoparticles entrapping water-insoluble photosensitizing anticancer drugs: a novel drug-carrier system for photodynamic therapy. J Am Chem Soc. 2003; 125:7860-7865.

14. Ohulchanskyy TY, Roy I, Goswami LN, et al. Organically modified silica nanoparticles with covalently incorporated photosensitizer for photodynamic therapy of cancer. Nano Lett. 2007;7:2835-2842.

15. Toole BP. Hyaluronan-CD44 interactions in cancer: paradoxes and possibilities. Cancer Res. 2009;15:7462-7468.

16. Karbownik MS, Nowak JZ. Hyaluronan: towards novel anti-cancer therapeutics. Pharmacol Rep. 2013;65:1056-1074.

17. Cui $\mathrm{X}, \mathrm{Xu} \mathrm{H}$, Zhou S, et al. Evaluation of angiogenic activities of hyaluronan oligosaccharides of defined minimum size. Life Sci. 2009; 85:573-577.

18. Tang N, Du G, Wang N, Liu C, Hang H, Liang W. Improving penetration in tumors with nanoassemblies of phospholipids and doxorubicin. J Natl Cancer Inst. 2007;99:1004-1015.

19. Chen Z, Li Z, Lin Y, Yin M, Ren J, Qu X. Bioresponsive hyaluronic acid-capped mesoporous silica nanoparticles for targeted drug delivery. Chemistry. 2013;19:1778-1783.

20. Ramasamy T, Tran TH, Choi JY, et al. Layer-by-layer coated lipidpolymer hybrid nanoparticles designed for use in anticancer drug delivery. Carbohydr Polym. 2014;102:653-661.

21. Li YF, Xiao B, Tu SF, Wang YY, Zhang XL. Cultivation and identification of colon cancer stem cell-derived spheres from the Colo205 cell line. Braz J Med Biol Res. 2012;45:197-204.

22. Lee H, Lee K, Kim IK, Park TG. Synthesis, characterization, and in vivo diagnostic applications of hyaluronic acid immobilized gold nanoprobes. Biomaterials. 2008;29:4709-4718.
Journal Citation Reports/Science Edition, EMBase, Scopus and the Elsevier Bibliographic databases. The manuscript management system is completely online and includes a very quick and fair peer-review system, which is all easy to use. Visit http://www.dovepress.com/ testimonials.php to read real quotes from published authors. 